\title{
Georg von Neumayer (1826-1909) \\ und die internationale Entwicklung der Geophysik
}

\section{Teil: Erdmagnetismus}

von Wilfried Schröder und Karl Heinrich Wiederkehr

\section{Zusammenfassung}

Im ersten Teil unserer Studie ( siehe Gesnerus 49, Part 1, S.45-62) haben wir die grossen Verdienste Neumayers um die Entwicklung der Meteorologie in aller Welt hervorgehoben. Im folgenden befassen wir uns, im Anschluss daran, mit Neumayers Bemühungen um die Erforschung des Erdmagnetismus. Auch auf diesem Feld war er Forscher und Denker, Anreger und Koordinator in einem.

\section{Erdmagnetische Landesvermessungen}

Während seiner Assistententätigkeit bei Johann von Lamont (1805-1879), Leiter der Sternwarte bei Bogenhausen (München) und einer der führenden Geomagnetiker nach der Ära des «Göttinger Magnetischen Vereins», wurde der Erdmagnetismus Georg Neumayers erste und später auch seine grosse Liebe. Ganz im Fahrwasser seines Lehrers führte er Mitte der 50er Jahre eine magnetische Vermessung der Rheinpfalz durch. Die Ergebnisse machte er allerdings erst später als Ruheständler bekannt ${ }^{40}$. In den von ihm ins Leben gerufenen Hydrographischen Mitteilungen von 1873 (ab 1875 Annalen der Hydrographie und maritimen Meteorologie) veröffentlichte er Beobachtungen von neun Orten Norddeutschlands, die dem Entwurf einer seiner ersten magnetischen Karten Mitteleuropas zugrunde lagen ${ }^{41}$. Von 1858 bis 1864 beobachtete G. Neumayer regelmässig am Flagstaff-Observatorium die erdmagnetischen Elemente und machte noch eine magnetische Aufnahme der damaligen englischen Kolonie Victoria ${ }^{42}$. Wenn auch magnetische Vermessungen in Norddeutschland nicht zu dem Aufgabenbereich der Seewarte gehörten, so wurden von ihr doch solche Projekte gefördert und die Publikationsorgane (Annalen der Hydrographie usw., Archiv der Deutschen Seewarte und Meteorologische Zeitschrift) zur Verfügung gestellt. Hierzu seien nur die 
Namen Scharper, Eschenhagen und Duderstadt genannt. Bevor G. Neumayer von Australien nach Europa zurückkehrte, bat ihn Edward Sabine als Präsident der Royal Society in London, das Ross-Observatorium und seine Umgebung in Hobarton (Tasmanien) magnetisch zu vermessen. Neumayers Bericht (in englischer Sprache) wurde nicht veröffentlicht; aber im «HannBand» der Meteorologischen Zeitschrift (zum 40. Redaktionsjubiläum, 1906) schildert uns Neumayer ausführlich dieses Unternehmen der australischen Pionierzeit ${ }^{43}$. Auf dem Deutschen Geographentag in Wien 1891 erläuterte G. Neumayer nochmals eingehend das methodische Vorgehen bei magnetischen Landesaufnahmen und deren Bedeutung ${ }^{44}$.

\section{Kompensation des Schiffskompasses}

Im Jahre 1871 reiste Neumayer nach Berlin, um für seinen Plan einer Zentralanstalt mariner Wissenschaften zu werben. Vor der dortigen «Gesellschaft für Erdkunde» hielt er auch einen Vortrag über die Störung des Kompasses auf eisernen Schiffen ${ }^{45}$. Um die Mitte des vergangenen Jahrhunderts hatte das eiserne Schiff zum Teil schon das hölzerne Handelsschiff verdrängt, und bald darauf ging man auch in den Kriegsmarinen zum Bau von Panzerschiffen über. Wissenschaftler, wie P.Barlow, A.Smith und G. B. Airy, hatten in England das Problem der Kompassablenkung angepackt ${ }^{46}$. Mit der Vergrösserung der deutschen Handelsflotte und den wachsenden Seeinteressen des Kaiserreiches musste man sich auch in Berlin mit diesem Problem auseinandersetzen. Albrecht von Stosch, Chef der Admiralität und mit dem Aufbau der Kaiserlichen Marine betraut, wurde auf Georg Neumayer aufmerksam und holte ihn als Hydrographen in sein Amt. Aber auch Neumayers weiterreichende wissenschaftliche Interessen für Erdmagnetismus und solarterrestrische Beziehungen ebneten ihm den Weg zu dieser einflussreichen Stelle. Wilhelm Foerster (1832-1921), Direktor der Berliner Sternwarte und als einer der Organisatoren des Wissenschaftsbetriebs in der Hauptstadt recht einflussreich, beschrieb in seinen Lebenserinnerungen, wie sehr er sich bei von Stosch für Neumayer eingesetzt hatte ${ }^{47}$. Foerster wollte in jenen Jahren ein «Sonnenobservatorium» errichten, das allerdings bald als «Astrophysikalisches Observatorium» konzipiert wurde ${ }^{48}$. In Neumayer fand er dabei einen kompetenten Mitstreiter. In den 50er und 60er Jahren hatte man den Einfluss der Sonne auf erdmagnetische 
Vorgänge erkannt (11jährige Periode in der Sonnenfleckenhäufigkeit und den erdmagnetischen Schwankungen, entdeckt durch E. Sabine, R. Wolf und J.-A.Gautier, parallel dazu die Polarlichthäufigkeit, nachgewiesen von H. Fritz 1862, und weitere Hinweise auf die Bedeutung der Solarphysik von K. F.Zöllner 1871) ${ }^{49}$.

Die Aufstellung eines Kompasses an Bord und seine Kompensation fiel der zweiten Abteilung der Deutschen Seewarte zu. Sie trug aber auch Sorge für eine Weiterentwicklung der Deviationslehre und gab der Technik und Industrie Anregungen zu Verbesserungen. Die neuerbaute und 1881 fertiggestellte Seewarte besass mit dem «magnetischen Pavillon» ein besonderes Gebäude, in dem die magnetischen Eigenschaften von Eisen- und Stahlsorten ermittelt wurden ${ }^{50}$. Regelmässige Beobachtungen der magnetischen Elemente wollte die Seewarte nicht durchführen, obwohl Instrumente wie der grosse magnetische Theodolit von Lamont, ein Intensitätsmessgerät nach Meyerstein und verschiedene Inklinatorien zur Verfügung standen. Wohl stellte man in den ersten Jahren Messungen an, und sogar absolute, aber die sich ausbreitende Grossstadt mit ihren elektrischen Anlagen setzte dem bald ein Ende. Das Observatorium der Kaiserlichen Marine in Wilhelmshaven nahm gewissermassen stellvertretend für die Seewarte diese Aufgabe wahr. Noch in seiner Zeit als Hydrograph hatte Neumayer 1873 den magnetisch günstigen Standort für das zu errichtende Observatorium bestimmt. Auf seinen Vorschlag hin wurde dann Carl Börgen, der an der Leipziger Sternwarte bei C. Bruhns Assistent war, 1874 zum Leiter des Instituts ernannt. Als Beleg dafür, wie sehr sich Börgen mit Neumayer verbunden fühlte, mag eine Stelle aus seinem Brief vom 29. Nov. 1873 zitiert werden ${ }^{51}$ :

«Ich weiss, und es ist eine grosse Freude für mich, dass ich Ihre Freundschaft mir erworben habe, und kann Ihnen meinen Dank dafür wohl nicht besser abtragen, als [...] Ihnen so viel in meinen Kräften steht, beizustehen in der Erstrebung der Ziele, die Sie sich gesetzt haben.»

\section{Erdmagnetische Untersuchungen im ersten Polarjahr}

Nachdem das Marineobservatorium mit photographisch-registrierenden Magnetometern, den ersten in Deutschland, ausgerüstet worden war, konnte es sich im «Polarjahr» 1882/83 in umfassender Weise beteiligen, und die 
deutsche Polarkommission übertrug C. Börgen die Bearbeitung der magnetischen Beobachtungen der deutschen Stationen ${ }^{52}$. Eschenhagen, Mitarbeiter am Observatorium in Wilhelmshaven, berichtete 1887 auf dem 7. Deutschen Geographentag in Karlsruhe über einige Resultate aus den erdmagnetischen Beobachtungen im Polarjahr ${ }^{53}$. Später trug auch Börgen darüber auf dem Meteorologenkongress in Chicago 1893 während der dort stattfindenden Weltausstellung (World's Columbian Exposition) vor ${ }^{54}$. Einen Überblick über die «neuesten erdmagnetischen Messungen in den Polarregionen» (Nansen, Borchgrevink, De Gerlache) gab G. Neumayer auf der Versammlung Deutscher Naturforscher und Ärzte in Aachen $1900^{55}$. Das Wilhelmshavener Observatorium nahm trotz anderer ihm zugewiesener Aufgaben in Meteorologie, Hydrologie und im Zeitdienst bis 1890 fast die Stelle einer magnetischen Zentralanstalt ein ${ }^{56}$. Das neugegründete magnetische Observatorium in Potsdam übernahm dann unter der Leitung von Eschenhagen diese Rolle.

Bei der Durchsicht des Konvoluts von Briefen, Briefentwürfen und anderen Papieren zum ersten «Polarjahr» - darunter befinden sich auch Schriftstücke aus Potsdam und Göttingen - ahnt man, welch' riesige Organisationsarbeit von Neumayer und den Mitarbeitern in der Seewarte zu bewältigen war ${ }^{57}$. Am Astrophysikalischen Observatorium in Potsdam war insbesondere H.K. Vogel an spektroskopischen Untersuchungen des Nordlichts interessiert. Die gelb-grüne Linie, die man damals noch keinem irdischen Stoff zuordnen konnte, gab besondere Rätsel auf. Auch E.Goldstein, ebenfalls Mitarbeiter an diesem Institut, wo er seine Experimente zu den Kathodenund Kanalstrahlen durchführte, beschäftigte sich mit den Polarlichtlinien. Über die Ursache und die physikalischen Vorgänge bei dieser farbenprächtigen Naturerscheinung wusste man damals noch recht wenig, nur soviel, dass die Erscheinung mit der Sonne zusammenhängt, und man vermutete elektrische Entladungen. Auch die Erdströme, die mit den Polarlichtern zusammen beobachtet wurden, sollten gemessen werden. Der Berliner Physiker G. R. Kirchhoff hatte dazu eine umfangreiche Anleitung gegeben. Die Ergebnisse der Polarlicht- und Erdstrom-Beobachtungen enttäuschten oder waren sogar ein Fehlschlag. Dass Nord- und Südlicht gleichzeitig auftreten können, hatte Georg Neumayer schon 1859 in Melbourne festgestellt, und während der Jahre in Australien auch die schwachen, aber doch regelmässigen erdmagnetischen Schwankungen, die mit dem scheinbaren Umlauf des Mondes verknüpft sind (lunare Variationen) ${ }^{58}$.

Dass das Gauss-Observatorium in Göttingen am vollen Programm des ersten Polarjahres unter Ernst Schering und seinem jüngeren Bruder Karl 
teilnehmen sollte, erschien selbstverständlich. Das Ministerium in Berlin behandelte in jenen Jahren allerdings die berühmte Forschungsstätte wie auch die ganze Universität in finanzieller Hinsicht etwas stiefmütterlich. Der Kurator von Warnstedt, den die preussische Regierung nach der Annektion des Königreichs Hannover 1866 im Amt belassen hatte, beklagte sich bitter darüber ${ }^{59}$. Durch das Eintreten Georg Neumayers und seinen Hinweis auf Göttingens berühmte Tradition wurden von Berlin Mittel für eine Erweiterung des Gauss-Observatoriums gewährt, wie aus Briefen E. Scherings und Briefentwürfen Neumayers hervorgeht ${ }^{60}$.

Dass die vierte und abschliessende Tagung der Internationalen Polarkommission 1884 in Wien stattfand, war Georg Neumayer zu verdanken. In seinem Schreiben an den Präsidenten der Kommission H. Wild in St. Petersburg heisst es:

«Als Ort der Zusammenkunft erscheint mir Wien sehr passend gewählt werden [zu] können, da wir dadurch die Verehrung und Dankbarkeit, die wir dem edlen Grafen Wilczek und den Mühen unseres verstorbenen Kollegen Weyprecht schulden, einen beredten Ausdruck geben können.»

In seinem Brief vom 8. Nov. 1883 antwortet Wild:

«[...] ich danke Ihnen sehr, dass Sie [...] durch einen bestimmten Vorschlag, auf den ich bis dahin Seitens der Mitglieder der Commission vergeblich wartete, die Initiative ergriffen haben.»

Einen weiteren Beitrag zu erfolgreicher Polarforschung lieferte Neumayer auf dem Gebiet der Konstruktion magnetischer Messgeräte. S. Scott-Hansen benutzte auf der sensationellen Driftfahrt des Norwegers Fridtjof Nansen mit der «Fram» 1893-1896 magnetische Instrumente, die nach Angaben Neumayers von C. Bamberg gebaut worden waren und sich bestens bewähr$\operatorname{ten}^{61}$.

Welche Kompetenz in Fragen der Polarforschungen und des Erdmagnetismus viele Zeitgenossen Neumayer zuerkannten, geht aus den Worten Roald Amundsens (1910 erreichte er als Erster den Südpol) in seinem Buch Die Nordwest-Passage (1907) hervor. Vor seiner Polarfahrt mit der Gjöa suchte Amundsen Rat und Unterstützung bei G. Neumayer. Er wollte den Magnetpol in der Arktis erreichen und seinen Ort bestimmen. Auf der Seewarte und nach dem Vorschlag Neumayers auch bei C. Börgen in Wilhelmshaven und bei A.Schmidt in Potsdam liess sich Amundsen mit den neuesten magnetischen Messgeräten und Messmethoden vertraut machen ${ }^{62}$. 


\section{Neuberechnung der Konstanten und Modifikation der Gaussschen Theorie durch A. Schmidt}

Georg Neumayer befasste sich auch theoretisch mit dem Erdmagnetismus. Zusammen mit H. Petersen führte er eine Wiederberechnung der 24 Gaussschen Konstanten der Kugelfunktionen durch, um sie dem aktuellen magnetischen Zustand der Erde anzupassen. Im genauen Anschluss an den Rechnungsgang von Gauss, nur mit Hinzuziehen des auf seiner Weltreise (18281830) gewonnenen Beobachtungsmaterials, hatte A.Erman schon mit H. Petersen für die Epoche 1829 erneut die Konstanten berechnet ${ }^{63}$. Neumayer stand ein viel umfangreicheres Datenmaterial zur Verfügung, darunter das von den Expeditionen der deutschen Korvette «Gazelle» (1874-1876) und dem englischen Forschungsschiff «Challenger», vor allem aber auch vom ersten Polarjahr. Alle Werte wurden in absolutes Mass umgerechnet, auf das Jahr 1885, 0 reduziert und hieraus dann Karten für den Physikalischen Atlas von H.Berghaus entworfen. Zur Berechnung der Konstanten wurden Punkte ausgewählt, daraus über die Kugelfunktionen die erdmagnetischen Elemente gewonnen und dann wieder mit den tatsächlichen Beobachtungen verglichen. Wie Neumayer auf dem 8. Deutschen Geographentag in Berlin 1889 mitteilte, war das Ergebnis wenig befriedigend ${ }^{64}$. Die sich anschliessenden Diskussionen ergaben, dass die Gausssche Theorie dringend einer Erweiterung bedurfte. A. Schmidt nahm diese Anregung auf und schlug mit seiner Modifizierung der Gaussschen Theorie einen in mehrfacher Hinsicht anderen Weg ein. Aufgrund des zur Verfügung stehenden dürftigen Materials hatte Gauss den Sitz des Erdmagnetismus im Inneren der Erde angenommen und negierte eine äussere, also ausserhalb liegende Ursache, obwohl er eine solche, in geringem Masse wirkende, durchaus im Bereich des Möglichen hielt und sogar von elektrischen Strömen in der Atmosphäre sprach ${ }^{65}$. Gauss nahm ein Potential an und führte die Rechnungen in vereinfachter Weise durch. Die Rechtfertigung für seine Annahme erhielt er nachträglich durch die ihm hinreichend erscheinende Übereinstimmung von theoretisch sich ergebenden und tatsächlich gemessenen Werten.

«Er [Gauss] zeigte aber auch», schrieb A. Schmidt in seinem Artikel «Erdmagnetismus» in der Encyklopädie der Mathematischen Wissenschaften, wie beim Verzicht auf diese Annahmen zu verfahren sei, und fügte eine Reihe weiterer bedeutsamer programmatischer Darlegungen über die künftige Ausgestaltung der Theorie und ihre Anwendung auf die verschiedenen Erscheinungen, insbesondere die Variationen, hinzu ${ }^{66} . »$

Innere und äussere Ursachen des Erdmagnetismus können nach Gauss 
getrennt werden. In seiner grundlegenden Arbeit «Mathematische Entwicklung zur allgemeinen Theorie des Erdmagnetismus», die in dem Sammelwerk Aus dem Archiv der Deutschen Seewarte 1889 erschien, entwickelte A. Schmidt jede Komponente für sich und berücksichtigte auch die Abplattung der Erde ${ }^{67}$. Die in weiteren Arbeiten durchgeführten numerischen Berechnungen - sie beruhten auf dem Neumayerschen Material - ergaben, dass ein geringer Anteil des Erdmagnetismus potentiallos ist und von elektrischen Strömen herrühren muss, die von der Atmosphäre in die Erde fliessen ${ }^{68}$.

In seinem Buch Auf zum Südpol, das selbstbiographische Züge trägt, glaubt Neumayer, dass hauptsächlich er den damaligen Gymnasiallehrer in Gotha, Adolf Schmidt, dazu bewogen habe, seine ganze Kraft der Erforschung des Erdmagnetismus zu widmen ${ }^{69}$. Und als mit dem Tode Eschenhagens 1901 die Stelle des Leiters am Potsdamer Magnetischen Observatorium frei wurde, setzte er sich vorbehaltlos für seinen Freund ein. In einem Brief an W.v. Bezold schrieb Neumayer ${ }^{70}$ :

«Bei $[\operatorname{der}][\ldots]$ besonderen Begabung [Eschenhagens] für die Construktion und Untersuchung von magnetischen Apparaten dürfte in der That ein entsprechender Ersatz nicht so schnell zu beschaffen sein. Von den beiden Gelehrten, die Sie für die durch den Tod Eschenhagens erledigte Stelle in's Auge fassten, verdient meines Erachtens Herr Prof. Schmidt in erster Linie eine Berücksichtigung, da seine hohe Begabung und seine Leistungen auf dem theoretischen Gebiet des Erdmagnetismus ihm ein gewisses Anrecht erworben haben.»

1902 übernahm dann A. Schmidt dieses Amt, und zur grossen Überraschung seiner Kollegen erwies er sich auch als hervorragender Praktiker und Konstrukteur magnetischer Messgeräte; man denke nur an seine Feldwaage, die heute noch im Gebrauch ist. Bald gehörte A.Schmidt zu den führenden Erdmagnetikern der ersten Hälfte unseres Jahrhunderts.

A. Schmidt zog bei seinen numerischen Berechnungen sogar Funktionen der sechsten Ordnung heran (48 Glieder). Noch weiter ging H. Fritsche; er setzte die Entwicklung bis zu den Funktionen siebenter Ordnung fort und erhielt so in den Reihen 63 Glieder ${ }^{71}$. Auch Fritsche legte Punkte aus Neumayers Karten für 1885 zugrunde. J. C. Adams errechnete ebenfalls die Konstanten für das Jahr 1880 (postum veröffentlicht 1898) ${ }^{72}$. Die von Neumayer-Petersen, Fritsche, Adams und Schmidt gefundenen Koeffizienten stimmen in den ersten massgebenden Zahlenwerten erstaunlich gut überein, obwohl Adams und Neumayer-Petersen sich auf unabhängige Materialsammlungen und Säkularreduktionen stützten. Eine bessere An- 
schmiegung von rückberechneten und beobachteten Elementen wurde damit allerdings auch nicht erreicht, der Erdmagnetismus ist doch von komplexerer Natur.

Über die physikalischen Ursachen des Erdmagnetismus und über die genaueren Vorgänge bei den Variationen tappte man zu Lebzeiten Neumayers noch fast völlig im dunkeln. Einen Lichtblick brachten Arthur Schusters Arbeiten zur täglichen Variation (1889). Angeregt durch einen Lexikonartikel von Balfour Stewart (1882) konnte er mit seiner Dynamotheorie - sie arbeitete mit induzierten Stromsystemen in der Atmosphäre und im Erdkörper - zum erstenmal diese Erscheinungen verständlich machen ${ }^{73}$.

\section{Erdmagnetische Karten}

Auf das Kartenwerk Georg Neumayers, das als IV.Abteilung des Physikalischen Atlas von Heinrich Berghaus in Gotha 1892 erschien, soll nochmals näher eingegangen werden. Der Atlas des Erdmagnetismus hat fünf kolorierte Hauptkarten und 20 Nebenkarten. Die Hauptkarten, die sich auf das Jahr 1885, 0 beziehen, stellen das Potential, die Isogonen, die Isoklinen und die Linien gleicher Horizontalintensität (Isodynamen im CGS-System) dar.

Die grösseren Karten sind Darstellungen in Mercatorprojektion, die Polargebiete sind auf Nebenkarten in Polarprojektion abgebildet. Unter den 20 Nebendarstellungen sind auch historische Karten, so z. B. nach Hansteen, Sabine, Gauss und Weber. Besondere Aufmerksamkeit schenkt Neumayer den Säkularveränderungen; hierin sieht er einen Schlüssel für zukünftige tragfähige Theorien. Die 5. Hauptkarte stellt die Änderung der magnetischen Deklination von 1600-1858 dar, und die 4 Nebenkarten die Isogonen für die verschiedenen Jahrhunderte. Der 1. Hauptkarte ist als Nebenkarte die Säkularänderung der Deklination von 1870-1890 beigegeben ${ }^{74}$. In einer 17 grossformatige Seiten umfassenden Einleitung legt Neumayer Rechenschaft ab über die Herkunft seines Datenmaterials und die Interpolationsmethoden, äussert sich über die wenig befriedigende Berechnung mit Hilfe der Gaussschen Konstanten und gibt einen Einblick in den damaligen Forschungsstand.

«Die Ergründung des Wesens der magnetischen Kraftäusserung der Erde und deren Zusammenhang mit kosmischen Vorgängen», schrieb er, «liegt nahezu noch unerschlossen vor uns.» 
Noch in seinen letzten Lebensjahren gab G. Neumayer magnetische Karten heraus, die den Stand um 1900 erfassten; auch eine Karte für die Säkularänderung (in Minuten pro Jahr) zu der Zeitspanne 1890-1900 ist darunter. L. A. Bauer nahm sie in seine U.S. Magnetic Declination Tables and Isogonic Charts for 1902 mit auf, und für die von Bauer herausgegebene Weltzeitschrift Terrestrial Magnetism and Atmospheric Electricity (1901) fertigte Neumayer hierzu eine kartographische Tabelle an ${ }^{75}$.

Karl Schering schrieb in seinem 3. Artikel «Über die Fortschritte unserer Kenntnis vom Magnetismus der Erde» im Geographischen Jahrbuch (1894):

«[Auf wissenschaftlichen Tagungen] [...] ist G. Neumayer unermüdlich thätig, das Interesse an erdmagnetischen Forschungen in weiten Kreisen zu wecken und rege zu halten.»

In sechs Artikeln, die im Abstand von ein paar Jahren in dem Geographischen Jahrbuch aufeinander folgten, gab Schering jeweils einen Überblick über alles Neue und Wissenswerte auf diesem Gebiet ${ }^{76}$. Auch hier half G. Neumayer mit seinen Literaturkenntnissen.

«Herr Professor Karl Schering hatte mir schon vor längerer Zeit mitgetheilt, dass er für das Jahrbuch einen Artikel über magnetische Forschungen zu verfassen hatte», liest man in einem Brief an den befreundeten H. Wagner in Göttingen, «und ersuchte mich um einige Materialien, die ich ihm zugehen liess. Hoffentlich wird dieser Überblick dazu beitragen, dass man nun auch in Deutschland mit neuem [?] [...] Interesse diesen lange recht vernachlässigten Zweig des menschlichen Wissens verfolgt ${ }^{77}$.»

Schon 1901 wies Neumayer auf der Versammlung Deutscher Naturforscher und Ärzte in Hamburg während einer Sitzung in der Seewarte auf die Bedeutung der Arbeiten Emil Wiecherts in der Seismik hin. Hier zeigt sich sein Gespür für die spätere Entwicklung in der Geomagnetik ${ }^{78}$.

Unsere Betrachtungen um die Verdienste Georg Neumayers bei der Einführung der synoptischen Meteorologie in Deutschland, ausgehend von der Maritimen und Küsten-Meteorologie, seine besonderen Beziehungen zu Wien, sein Ringen um Fortschritte in der Kenntnis vom Erdmagnetismus und sein Wirken in Anwendung und Praxis wollen wir mit einem Zitat W. Köppens beschliessen. In dem Nachruf auf Neumayer heisst es ${ }^{79}$ :

«Diese Zeit des Neuschaffens bot dem ausgesprochenen organisatorischen Talent Neumayers das richtige Fahrwasser. Denn er gehörte weniger zu jenen Gelehrten, die in der Stille der Studierstube Gesetze finden oder im Laboratorium durch Experiment und Beobachtung neue Tatsachen suchen, als zu denen, die nach grossen Gesichtspunkten die wissenschaftliche Arbeit organisieren und durch ihre Schöpfungen sich einen dauernden Platz in der Geschichte der Wissenschaft sichern.» 


\section{Quellen und Literatur}

\section{Teil II}

40 G. Neumayer: Eine erdmagnetische Vermessung der bayerischen Rheinpfalz 1855/56, Mitteil. der Pollichia, Bad Dürkheim 1905.

41 Hydrographische Mittheilungen 1.Jg. 1873, S.5-7 und 282-287 mit Karte.

42 Siehe Anm. 5 in Gesnerus 49, Part I, S.59.

43 G. Neumayer: Die geologisch-magnetischen Verhältnisse des einstigen erdmagnetischen Observatoriums in Hobarton (Tasmanien). In: Meteorologische Zeitschrift, Hann-Band, redig. von J. M. Pernter und G. Hellmann, Braunschweig 1906, S. 67-81.

44 G. Neumayer: Über die Bedeutung und Ziele erdmagnetischer Landesvermessungen. In: Verhandlungen des Neunten Deutschen Geographentages zu Wien 1891. Berlin 1891, S.11-27.

45 Zeitschrift der Gesellschaft für Erdkunde zu Berlin. Hrsg. von W. Koner. 7. Bd. Berlin 1872, S. 93-95.

46 H.Meldau: Nautik. In: Encyklopädie der mathematischen Wissenschaften mit Einschluss ihrer Anwendungen. 6.Bd. 1. Teil. Hrsg. von Ph. Furtwängler und E. Wiechert. Leipzig 1906-1925. Geodäsie S. 320. - G. Neumayer: Die Thätigkeit der Deutschen Seewarte während der ersten 12 Jahre ihres Bestehens (1875-1886). Hamburg 1887, S. 14.

47 W. Foerster: Lebenserinnerungen und Lebenshoffnungen. Berlin 1911, S.130-133.

48 D.B.Herrmann: Zur Vorgeschichte des Astrophysikalischen Observatoriums Potsdam (1865 bis 1874). In: Astronomische Nachrichten Bd.296. Berlin 1975, S.245-259. W.Schröder: Wilhelm Foerster und die Entwicklung der Solarterrestrischen Physik. In: Die Sterne 59 (1983) S.348-352 - K.-H.Tiemann: Die organisatorischen Verdienste des Astronomen W.J.Foerster (1832-1921) auf dem Gebiet der Geowissenschaften. In: W. Schröder (Hrsg.), Advances in Geosciences. Bremen-Rönnebeck 1990, S.319-333.

49 W.Schröder: Das Phänomen des Polarlichts. Darmstadt 1984.- Ders.: Theorienwandel des Polarlichts. In: Gerlands Beitr. Geophysik 97. Leipzig 1988, S. 534-548.

50 Aus dem Archiv der Deutschen Seewarte, 7.Jg. 1884. Beschreibung der Zentralstelle in Hamburg. Hamburg 1885. Tafel 23.

51 Deutsches Museum München, Bibliothek, Urkunden- und Handschriften-Sammlung. 84 Briefe von K. Börgen an G. Neumayer 1873-84.

52 Siehe Anm. 36 in Gesnerus 49, Part I, S. 61.

53 M. Eschenhagen: Einige Resultate der erdmagnetischen Stationen im System der internationalen Polarforschung. In: Verhandlungen des 7. Deutschen Geographentages zu Karlsruhe 1887. Berlin 1887, S. 141-151.

54 C. Börgen: The International Polar-Expeditions 1882-83. In: Report of the Chicago Meteorological Congress August 1883. Part II pp.469-485. - Siehe dazu auch Robert D. Multhauf and Gregory Good: A Brief History of Geomagnetism and A Catalog of the Collections of the National Museum of American History. Washington 1987, S. 36.

55 Kurzer Auszug von G. Neumayers Vortrag in: Met. Zs. 1901, S. 179.

56 D. Voppel: Hundert Jahre Erdmagnetischer Dienst in Norddeutschland. In: H.Birett, K. Helbig, W. Kertz und U.Schmucker (Hrsg.), Festschrift zur 50jährigen Wiederkehr der Gründung der Deutschen Geophysikalischen Gesellschaft. Berlin-Heidelberg-New York 1974.

57 Im Besitze der Bibliothek im Bundesamt für Seeschiffahrt und Hydrographie in Hamburg. 
58 G. Neumayer: Auf zum Südpol. Berlin 1901, S.216. G. N. untersuchte die Abhängigkeit von der Deklination des Mondes. Philos. Trans. 1867, S.506. - Siehe dazu auch A.Schmidt: Erdmagnetismus. In: Encyklopädie der math. Wiss. 6. Bd. 1.T. (s. bei 46). Geophysik S. 267-396 (abgeschlossen 1917). Hier S.338.

59 G.v. Selle: Die Georg-August-Universität zu Göttingen. Göttingen 1937, S. 317.

60 E. und K.Schering: Beobachtungen in Gauss' erdmagnetischem Observatorium der Kgl. Univ. Göttingen während der Polarexpeditionen 1882 und 1883. In: E.Schering, Gesammelte Werke. Hrsg. von R. Haussner und K. Schering. 2 Bde. Berlin 1902 und 1909. Hier 2. Bd. S. 107-144 und 468-470.

61 K. Schering: Bericht über die Fortschritte unserer Kenntnisse vom Magnetismus der Erde (VI, 1899-1904). Geographisches Jahrbuch 28. Bd. (1905). Hrsg. von H. Wagner. S. 359. G. Neumayer: Neues auf dem Gebiete des Welt- und Erdmagnetismus. Gedruckter Vortrag der Pollichia, Bad Dürkheim 1900, S.13.

62 R.Amundsen: Die Nordwest-Passage. Meine Polfahrt auf der Gjöa 1903-1907. München 1907, S.4. - Ebenso Edouard Calic: Kapitän Amundsen. Leipzig 1966, S.67-69.

63 A. Erman und H.Petersen: Die Grundlagen der Gaussischen Theorie und die Erscheinungen des Erdmagnetismus im Jahre 1829. Berlin 1874.

64 G. Neumayer: Über das gegenwärtig vorliegende Material für erd- und weltmagnetische Forschung. In: Verh. des Achten Deutschen Geographentages zu Berlin 1889. Berlin 1889, S. 33-66.

65 C. F. Gauss: Allgemeine Theorie des Erdmagnetismus. In: Resultate aus den Beobachtungen des Magnetischen Vereins im Jahre 1838. Leipzig 1839, S. 50.

66 A.Schmidt: Erdmagnetismus. In: Encyklopädie der math. Wiss. 6. Bd. 1.T. (s. Anm. 58) S. 365 und 366 .

67 A. Schmidt: Mathematische Entwicklungen zur allgemeinen Theorie des Erdmagnetismus. In: Aus zum Archiv der Deutschen Seewarte. 12. Jg. (1889) Nr.3.

68 A. Schmidt: Mitteilungen über eine neue Berechnung des erdmagnetischen Potentials. In: Abhandlungen der mathematisch-physikalischen Klasse der kgl. bayerischen Akademie der Wiss. Bd. 19, München 1895.- Ders. : Der magnetische Zustand der Erde zur Epoche 1885, 0. In: Aus dem Archiv der Deutschen Seewarte, 21. Jg. (1898) Nr.2.

69 G. Neumayer: Auf dem Südpol, Berlin 1901, S.418.-Die zahlreichen Briefe Neumayers im handschriftlichen Nachlass von A.Schmidt im Besitze der Forschungsbibliothek Gotha müssten dies weiter belegen. Siehe dazu Helmut Roob und Peter Schmidt: Adolf Schmidt 1860-1944. Gotha 1985.

70 Brief G. Neumayers an W.v. Bezold vom 21. Nov. 1901. Kopie im Besitze der Pollichia im Pfalz-Museum für Naturkunde in Bad Dürkheim.

71 H. Fritsche: Über die Bestimmung der Koeffizienten der Gaussischen allgemeinen Theorie des Erdmagnetismus für das Jahr 1885 usw., St. Petersburg 1897.

72 A.Schmidt: Erdmagnetismus. In: Encyklopädie der Math.Wiss. VI. Bd. S.369. (Vgl. Anm. 46 n. 58).

73 Gregory A.Good: The Study of Geomagnetism in the Late 19th Century. In: Eos Vol. 69 (1988).

74 Zur Bedeutung der Säkularvariationen für physikalische Theorien siehe Stephen G. Brush and S.K. Bannerjee: Geomagnetic Secular Variation and Theories of the Earth's Interior. In: W.Schröder (Ed.), Past, present and future trends in geophysical research. BremenRönnebeck 1988, S.65-97. 
75 K. Schering: Bericht über die Fortschritte unserer Kenntnisse vom Magnetismus der Erde (VI, 1899-1904). In: Geogr. Jahrbuch 28. Bd. 1905, S. 295.

76 Geographisches Jahrbuch, hrsg. von H. Wagner, 13 (1889), 15 (1891), 17 (1894), 20 (1897), 23 (1900) und 28 (1905).

77 Brief G. Neumayers an Hermann Wagner vom 24.1.1889, im Besitze der Niedersächsischen Staats- und Universitätsbibliothek Göttingen. Wagner nahm an Fragen der Geophysik regen Anteil. Siehe dazu W.Schröder: Die Georgia Augusta und der Aufstieg der Geophysik zur exakten Naturwissenschaft. In: Informationen Universität Göttingen, Mai 1983, S. 9.

78 Annalen der Hydrographie usw., 31.Jg. (1903). Kleinere Mitteilungen, S.511. - Und G. Neumayer: Neues auf dem Gebiete des Welt- und Erdmagnetismus (s. Anm. 61), S. 7.

79 W. Köppen: Nachruf für G.v. Neumayer. In: Met.Zs. 1909, S. 404. 


\section{Summary}

\section{Georg von Neumayer (1826-1909) and international geophysics}

Part II : Geomagnetism

Neumayer was a prominent figure in the development of geophysics in the 19th century and in organizing a network of international cooperation in the new science. While in the $1^{\text {st }}$ part of their work (see Gesnerus 49, Part 1, p. 45-62), the authors considered Neumayer's merits in the field of meteorology, they analyse in this $2^{\text {nd }}$ part his contributions to the investigation of terrestrial magnetism. In this field too Neumayer was a research worker and a thinker as well as a stimulating coordinator. In 1892, he published, as part IV of Berghaus's Physikalischer Atlas, an excellent Atlas of geomagnetism.

\section{Résumé}

Georg von Neumayer (1826-1909) et le développement de la géophysique sur le plan international

\section{$2^{e}$ Partie: Magnétisme terrestre}

Dans la première partie de leur travail (voir Gesnerus 49, Part 1, p. 45-62), les auteurs ont mis en relief les mérites de Neumayer pour la météorologie. Dans cette $2^{\circ}$ partie, ils examinent sa contribution à une autre branche de la géophysique, l'investigation du magnétisme terrestre. Là aussi, Neumayer était chercheur, penseur et coordinateur stimulant à la fois. En 1892, il publia, comme 4 partie du Physikalischer Atlas de Berghaus, un excellent Atlas du magnétisme terrestre.

Dr. Wilfried Schröder

Hechelstr. 8

D-2820 Bremen-Rönnebeck

Dr. rer. nat. habil Karl-Heinrich Wiederkehr

Birkenau 24

D-2000 Hamburg 76 